\section{Mini invasive hemodynamic monitoring: from arterial pressure to cardiac output}

\author{
GIORGIO DELLA ROCCA • MAURIZIO CECCONI • \\ MARIA GABRIELLA COSTA
}

\author{
GIORGIO DELLA ROCCA $(\triangle) \bullet$ \\ MAURIZIO CECCONI • \\ MARIA GABRIELLA COSTA \\ Department of Anesthesia \\ and Intensive Care Medicine \\ Medical School of \\ University of Udine \\ P.le S. M. Misericordia, 15. \\ 33100 Udine, Italy \\ Phone: +390432 559501 \\ Fax: + 390432559512
}

E-mail: anestesia.segr@aoud.sanita.fvg.it

\begin{abstract}
To evaluate the Cardiac Output (CO) the standard invasive pulmonary artery catheter (PAC) is considered today the gold standard. The major criticism to the PAC is that its level of invasiveness is not supported by an improvement in patient's outcome. The interest to lesser and lesser invasive techniques is high. Therefore, the alternative techniques have been recently developed.

Cardiac Output can be monitored continuously by different devices that analyze the arterial waveform to track changes in stroke volume (SV) and CO. The analysis of the arterial pressure wave to determine cardiac output is classified as Pulse Contour analysis or Pulse Pressure Analysis. Starting from a similar principle three main devices are now available on the market, with different algorithms and features:

- PiCCO System (Pulsion Medical System, Munich, Germany)

- LiDCO ${ }^{T M}$ plus System (LidCO, Cambridge, UK)

- Flotrac technology and Vigileo Monitor (Edwards Lifesciences, Irvine, CA, USA).

The algorithm used by all these devices has been also implemented even with the analysis of the variation of stroke volume (SW) and of the pulse pressure (PPV). SW and PPV represent the variation of stroke volume and of the pulse pressure during the respiratory cycle. In sedated ventilated patients these indexes have proven to predict the response to a fluid challenge. A high variation (>10-12\%) identifies with good sensitivity and specificity responders and not responders.
\end{abstract}

Key words: cardiac output, arterial pressure, stroke volume variation, pulse pressure variation

The hemodynamic monitoring has been continuously updated from the standard invasive pulmonary artery catheter (PAC) and the routine arterial line to lesser and lesser invasive techniques. The major criticism to the PAC is that its level of invasiveness is not supported by an improvement in patient's outcome. Monitoring cardiac output is essential in hemodynamic patients, so new devices have been developed with the aim of monitoring circulation. Some of these methods are less invasive than the PAC, but still require the catheterization of blood vessels. Over this decreasing level of invasiveness one of the major problems still remains open: to define a clinical useful index of preload. Preload is the initial stretching of the myocyte before contraction. Unfortunately this stretching cannot be measured in vivo and therefore other methods have been developed to estimate preload in vivo. Preload can be considered the volume of blood at the end of diastole (after atrial contraction) in the ventricles. The knowledge of preload is important when treating hemodynamically unstable patients to identify which ones and how much they will require volume expansion: from this point on a pressure will generate a flow and vice versa. The challenge of the latest monitors is to evaluate the cardiac output simply form the systemic arterial pressure (AP) through a pulse pressure based algorithm.

Cardiac Output (CO) can be moni- 
tored continuously by different devices that analyze the arterial waveform to track changes in stroke volume (SV) and $\mathrm{CO}$. The analysis of the arterial pressure wave to determine cardiac output is classified as Pulse Contour analysis or Pulse Pressure Analysis. Starting from a similar principle three main devices are now available on the market, with different algorithms and features:

- PiCCO System (Pulsion Medical System, Munich, Germany)

- LiDCO ${ }^{T M}$ plus System (LidCO, Cambridge, UK)

- Flotrac technology and Vigileo Monitor (Edwards Lifesciences, Irvine, CA, USA).

Compliance describes the relationship that between Pressure and Volume in the arterial vessels. Knowing the compliance is essential to estimate the changes in volume (stroke volume) from changes in pressure.

$\mathrm{C}=\Delta \mathrm{V} / \Delta \mathrm{P}$

$\mathrm{C}=$ compliance, $\Delta \mathrm{V}=$ changes in volume, $\Delta \mathrm{P}=$ changes in pressure

Without knowing the compliance of the arterial tree, more precisely the compliance of the proximal aorta, it is not possible to understand which change in volume corresponds to a change in pressure. Compliance in the arterial tree is not a linear relationship and so the same change in pressure does not always reflect the same change in volume. As a general rule compliance is higher for lower pressure and lower for higher pressure. Another peculiarity of compliance is that it is not constant. The same arterial tree can behave differently in different situation and be characterized by different compliance. For example vasoconstriction during vasopressor therapy decreases compliance, vice versa vasodilation during vasodilator therapy or sepsis increases compliance.

As we mentioned before, the aorta is the arterial vessel of interest, because it is the part of the arterial tree that receives directly the stroke volume. There are three forces that contribute to how the proximal aorta is filled with blood at every heart beat:
- the force of injection of the blood by the pumping of the heart

- the opposing force dependent on the pulsatile inflow (impedance)

- the opposing force, dependant on the change in volume that we described as compliance.

When blood leaves the aorta there is also another force that opposes to the blood to flow peripherally. This is the peripheral resistance.

There is also another problem to solve: the recorded periphery arterial pressure recorded is dependent not only on the aortic arterial pressure, but it is the result of the reflection waves generated peripherally. Furthermore pressure waves are the ones recorded from the arterial transducer. If it is true that in the proximal aorta the flow wave and the pressure wave happen almost synchronously, peripherally pressure wave is transmitted about 20 times faster than the flow wave. Moreover, even if it is true that in proximal aorta the flow is almost pulsatile, blood flows peripherally both in systole and in diastole. A pulse pressure algorithm needs to take into consideration all these forces when transforming a pressure in a volume in order to track stroke volume.

Summarizing an ideal algorithm should:

- work independent of the arterial site from where pressure is monitored despite changes in waveform shape and pressure through the arterial tree from central to the periphery

- correct for non linear compliance and take account of individual variations in aortic characteristics giving so an absolute cardiac output

- be not affected by changes in vascular resistance causing changes in reflected wave augmentation of the arterial pressure

- not rely on identifying details of wave morphology

- be only minimally affected by the damping often seen in arterial lines.

The first algorithm used in clinical practice was the Wesseling algorithm in 1983. (1) His algorithm is based on the concept that the contour of the arterial pressure waveform is dependent from stroke volume, and that this can be estimated from the integral of the change in pressure over time, considering the interval between ends of diastole to end of systole (Asys). This is the first algorithm of pulse contour analysis used to determine cardiac output. From this point, new algorithms have been developed. (2)

PiCCO system. In clinical practice the first pulse contour method using a Wesseling based algorithm was PiCCO system (Pulsion Munich, Germany). PiCCO is a cardiac monitor that measures cardiac output and several volumes (intrathoracic blood volume ITBV, Global End Diastolic Volume GEDV, Extravascular Lung Water EVLW) via transpulmonary thermodilution. This thermodilution is used to calibrate the pulse pressure algorithm. The pulse pressure algorithm analyses both the systolic and the diastolic part of the arterial pressure to study and determine the non linear compliance, and the relation flow/pressure. (2)

This part of the process is called calibration and should be repeated every time there is a significant hemo-dynamic change. In order to perform a thermodiIution a central venous catheter needs to be in place. $15 \mathrm{~mL}$ of cold saline are injected through a sensor that detects temperature and time of the injection. A specialized arterial catheter (usually a femoral artery) detects the changes in blood temperature, producing a thermodilution curve. The same arterial catheter is used to monitor blood pressure and the arterial waveform is analysed by the pulse pressure algorithm. PiCCO continuous cardiac output had been studied and validated against pulmonary artery catheter (PAC) in several conditions and has proven to be a reliable device, requiring to be calibrated more often only in case of major hemodynamic changes.

The algorithm is implemented even with the analysis of the variation of stroke volume (SVV) and of the pulse pressure (PPV). SW and PPV represent the variation of stroke volume and of the pulse pressure during the respiratory cycle. In sedated ventilated patients 
these indexes have proven to predict the response to a fluid challenge. A high variation (>10-12\%) identifies with good sensitivity and specificity responders and not responders.

LiDCO TM plus system is a cardiac output monitor that measures cardiac output via lithium transpulmonary thermodilution. The LiDCO algorithm for continuous cardiac output monitoring is based on the hypothesis that the change in power in the system (arterial tree) during systole is proportional to the difference of the amount of blood entering the system (stroke volume) minus the amount of blood flowing out peripherally. It is based on the principle of conservation of mass/power and an assumption that following correction for compliance and calibration there is a linear relationship between net power and net flow. This algorithm defines which part of the "change in power" is determined by the stroke volume. When this is identified then cardiac output is derived. The calibration is obtained via Lithium dilution technique. In order to perform the calibration a dose of $0.3 \mathrm{mMol}$ of lithium is injected using either a central or a peripheral venous access. A sensor connected to an arterial line (there's no need of a specialized catheter) makes possible to generate a concentration-time curve and the cardiac output is then calculated. This value is then used to calibrate the pulse pressure algorithm. Further calibrations should be performed in case of major hemodynamic changes.

Continuous cardiac output of LiDCO has already been validated in a few studies. (3) This new algorithm has so far proven to be reliable in surgical and intensive care patients. LiDCO algorithm is implemented with the analysis of the variation of stroke volume (SV), of the pulse pressure (PPV) and of the systolic pressure (SPV). As discussed for PiCCO these indexes are useful predictors of fluid responsiveness. (4)

Flotrac (Edwards Lifescience, Irvine, CA, USA) is the name of the specific transducer incorporated in the moni-tor Vigileo. The most interesting cha-racteristic of this device is that it doesn't require to be calibrated and it needs just an arterial line to work. Compliance and resistance are derived from the analysis of the arterial waveform. The hypothesis is that in order to calculate the effects of compliance and peripheral resistance on flow all the necessary information can be obtained by the analysis of the arterial pressure waveform. Age, Weight and Sex of the patient are the only information that the clinician needs to insert into the Vigileo monitor. The transducer (Flotrac) can be connected to whatever arterial line. The algorithm recalculates continuously the compliance bypassing in this way the need of calibration. Vigileo algorithm is implemented with the analysis of the variation of stroke volume (SW) and pulse pressure (PPV). This algorithm is now under validation.

\section{Clinical use of pulse pressure based algorithms}

The more interesting feature of all these devices is that they are able to track changes of cardiac output in real time. Intermittent techniques are indeed not appropriate to measure changes when these happen in a very short interval (seconds, minutes). This is particularly useful when evaluating the effect of fluid challenge or inotrope administration on hemodynamics. When performing a fluid challenge recognizing an increase in SV (>10\%) identifies patients that benefit from fluids. All the presented devices have features that make them suitable to monitor in real time changes in cardiac output when a therapeutic intervention is applied.

For the devices in which it is possible, it is necessary to recalibrate the system after any main hemodynamic events. It is finally important to remind, for all three, the importance of the shape of the waveform, without any dumping, to have a correct evaluation of the $\mathrm{CO}$, SVV and PPV. (4)

The historical milestone represented by the PAC has not been deleted, but a new chapter of hemodynamic monitoring has been written due to the development of the above described mini invasive techniques.

\section{REFERENCES}

1. Jansen JR, Wesseling KH, Settels JJ, Schreuder JJ. Continuous cardiac output monitoring by pulse contour during cardiac surgery. Eur Heart J 1990;11:26-32.

2. Della Rocca G, Costa MG, Pompei L, Coccia C, Pietropaoli P. Continuous and intermittent cardiac output measurement: pulmonary artery catheter versus aortic transpulmonary technique. Br J Anaesth 2002;88:350-6.

3. Michard F, Teboul JL. Using heart-lung interactions to assess fluid responsiveness during mechanical ventilation. Crit Care 2000;4:282-9.

4. Hamilton TT, Huber LM, Jessen ME. PulseCO: a less-invasive method to monitor cardiac output from arterial pressure after cardiac surgery. Ann Thorac Surg 2002; 74:1408-12. 